\title{
MANGEMENT OF BLOCKCHAIN BASED DIGITAL ASSETS IN INDUSTRY AND SERVICES
}

\author{
Paweł SZEWCZYK \\ Banking University in Poznan, Faculty in Chorzow; pszewczyk@chorzow.wsb.pl, \\ ORCID: 0000-0003-4240-4532
}

Purpose: The technology industry has become incredibly dynamic and complex, so by utilizing the information technology (IT) framework in the approach to new technology, businesses can avoid confusion around the application of new trends since IT operations can deliver business value.

Design/methodology/approach: An overview of recent literature of some assets digitization concepts was applied. In particular the concept of digital twins was presented. Finally, applying the blockchain technology the security from hackers due to its encryption features may be provided.

Findings: The improvement of digital twins through blockchain technology is set to transform industries such as manufacturing, healthcare and beyond and, companies can work towards building their future business strategies.

Practical and social implications: The impact that digital twins can make is huge. Many are just now in development due to the complexity of their creation, but soon millions of things will have digital twins. And their usefulness and capabilities will continue to evolve. On the other hand blockchain technology has the potential to become the most relevant and capable way to generate and monitor digital twins due to the security and immutability of technology.

Originality/value A jet engine, a human heart, even an entire city can all have a digital twin that mirrors the same physical and biological properties as the real thing enabling three critical drivers: continuous evaluation; faster, cheaper prototyping and innovating at the limits (ethics).

Keywords: blockchain, digital assets, digital twins.

Category of the paper: Viewpoint, General review.

\section{Introduction}

As the world becomes more digital, companies are transforming to use technology more intelligently and more strategically. This transformation in the face of growing complexity is a huge challenge. By utilizing the information technology (IT) framework in the approach to new technology, businesses can avoid confusion around the application of new trends and 
distraction that could lead to inefficiency. The basic foundation of infrastructure, development, security, and data has evolved over time, but it continues to define IT operations that deliver business value (CompTIA.org: A Functional IT Framework, Report 2016). We are living in a digital economy. Every business today can see that technology has become a more critical part of their operations and their future success. In order to remain competitive, companies know they must invest in IT. But that knowledge is not enough; they also must know where those investments should be made. Simply investing more in existing IT tactics is not sufficient. Enterprise technology has gone through a major shift, and understanding that shift is a prerequisite for a winning game plan. Modern IT has expanded to more explicitly serve a dual purpose. Along with the tactical support work that continues to be a requirement, IT now has a role to play in directly driving strategic objectives. Customer acquisition happens on digital platforms. Brand awareness is built through social media. Market share is gained through omnichannel experiences. These initiatives are not achieved simply by building programs on top of technology, but by using technology as the primary mechanism for success. (CompTIA.org: Using Strategic IT for Competitive Advantage, Whitepaper 2018). Emerging technologies have become a key part of the discussion around modern digital organizations. Across the high-tech industry, the term "emerging technology" has become a catch-all descriptor for the futuristic advances that will drive the next wave of innovation, and there is a wealth of resources available for understanding the individual trends that fall under that broad umbrella. From artificial intelligence (AI) to quantum computing, there has been a hunger to find the next big thing. As part of the ongoing evolution towards more strategic thinking, IT leaders of the future will embrace new techniques and responsibilities that will drive greater value into the business (CompTIA.org: The Role of Emerging Technology in Digital Transformation, Whitepaper, 2020).

This general review paper covers those papers which provide an overview of some concepts and techniques concerning digital assets especially in the form of digital twins and presents opinions of different research circles on how, by applying the blockchain technology, the concept of digital twins can be improved. As a basic source of opinions served works of The European Blockchain Observatory and Forum which launched in February 2018, and which is "a European initiative to accelerate blockchain innovation and the development of the blockchain ecosystem within the EU and so help cement Europe's position as a global leader in this transformative new technology" (EU Blockchain Forum WG, 2018). 


\section{Digital assets}

The most basic description of digital assets may be found in the Thematic Report: Digital assets (Blockchain forum.eu: report 2020a, p. 7). "The concept of "digital assets" is not a new one, and does not inherently rely on blockchain. Digital assets, sometimes called tokens ${ }^{1}$, exist in the same way that electronic record-keeping systems do. They can be defined as a string of characters, often stored in a binary format, that represent values or rights that can be exercised within a specific context. For this reason, the term "digital assets" does not necessarily refer to assets issued and transferred on a blockchain, since this is a sub-group within the more generic definition of digital assets. Each digital asset is associated with one blockchain, on which transactions on such assets are stored within blocks. Digital assets can be programmed to serve many different functions. For example, they can represent a resource earned or produced within a sharing economy or environment (such as computing power units), rights (access, voting, etc.), identity or attestations of facts (driving licence, degree, etc.), physical assets (gold, commodities, etc.), securities (shares, debt instruments, etc.), or even property (such as a painting or real estate). Sometimes various functions overlap within the same token, making the asset difficult to place under a single heading". Thanks to blockchain, digital assets can be created by almost anyone with the technical know-how, a process generally referred to as "tokenisation", and can be distributed in a number of innovative ways. The technology also makes them easy to trade on secondary markets, but introduces new concepts and raises new challenges when it comes to asset custody. Thanks to smart contract technology running on blockchain (Blockchain forum.eu: report 2020, Blockchain terminology) digital assets can also be "programmed" - adding new capabilities that are not possible using traditional means of asset issuance and exchange. As such, digital assets can both reflect the traditional world of assets and represent something completely new in the world. This has ignited debate around how to categorise and legally qualify them. While public authorities in many jurisdictions have been examining these issues, today there is no globally recognised, binding taxonomy in use.

In the mentioned above EU Digital assets Thematic Report an informal working consensus has developed around the three basic digital asset categories of:

1. payment/exchange/currency tokens;

2. investment/security tokens; and

3. utility/consumption tokens. Existence of a number of hybrid tokens, which have features spanning more than one of these categories, shows, however, the difficulties that still remain in coming to an agreement on how digital assets should be classified.

\footnotetext{
${ }^{1}$ Tokens are a type of digital asset that can be tracked or transferred on a blockchain. Tokens are often used as a digital representation of assets like commodities, stocks and even physical products.
} 
Don Tapscott, head of the Blockchain Research Institute (BRI) and his co-workers (BRI, 2020) have done significant work defining models for blockchain governance and stewardship, and believe that the most robust form of stewardship is the multi-stakeholder non-state network, consisting of participants from the private sector, government, academia, and civil society at large. For a technology to achieve mass adoption and longevity, such networks must provide stewardship in several areas, such as the development of standards, policy, and knowledge, and the governance of changes to the technology.

In a recent BRI Big Idea White Paper (Tapscott, 2020) Tapscott observes that digital assets, sometimes called tokens, are poorly understood. That may be why they are used to describe a variety of things, some of which are contradictory. In order to help the understanding of the token economy a new standardization framework - the Token Taxonomy Initiative (TTI) was formed by many creators of the enterprise blockchain. It starts with token types and progresses to token properties, with a goal of enabling anyone-technologist, businessperson, or regulator - to understand what is happening and how to take part in token projects. "Standardization requires a commitment to collaborating with competitors, stewarding shared creations faithfully, and developing certification programs that make it easier for users to detect fakes and harder for unscrupulous designers to claim the use of standards while deviating from those standards".

The benefits of shared token standards are many: increased ease of use, improved interoperability, easier communication, and faster and more secure development of the token projects. In conclusion the author states (Tapscott, 2020, p. 21): "Ultimately, there is much work left to be done. The Token Taxonomy Framework is a fantastic base layer, but it won't solve the significant problems that the blockchain community faces overnight. To achieve its full potential, the TTF will require continued adoption, development, and improvement by a wide variety of actors".

Summing up, it is clear that the concept of digital assets is simple. Applying it in practice is harder. The problem with digital assets and blockchain ecosystems is that they are complex. It is not the technology but also all the aspects around it. Questions include: what is the core protocol, the consensus mechanisms, who are the participants, who are the promoters, what are the promises and expectations?

\section{Digital twins}

In January 2020 Purdy, M., Eitel-Porter, R., Krüger, R. and Deblaere, T. published in MIT Sloan Management Review (Purdy et al., 2020) a paper entitled: 'How Digital Twins Are Reinventing Innovation' with a subtitle: From faster and cheaper drug trials to fully "conscious" cities, digital replicas are changing the face and pace of innovation. The authors write that 
a digital twin - a virtual replica of an object, being, or system can be continuously updated with data from its physical counterpart.

Supported by an estimated 25 billion connected global sensors by 2021 (Gartner, 2018) ${ }^{2}$, digital twins will soon exist for millions of things. A jet engine, a human heart, even an entire city can all have a digital twin that mirrors the same physical and biological properties as the real thing. The implications are profound:

- real-time assessments and diagnostics much more precise than currently possible;

- repairs literally executed in the moment; and

- innovation that is faster, cheaper, and more radical.

Digital twins stand to change the innovation game by enabling three critical drivers (Purdy et al., 2020):

1. Continuous evaluation. Traditionally, most complex products could be fully analyzed, piece by piece, only twice during their lifetime - when they were created and when they were broken down at the end of their life cycle. Now that sensors can capture and continuously update the product's digital twin throughout its lifetime, manufacturers have a live window inside the product at all times.

2. Faster, cheaper prototyping. Digital twins can dramatically lessen the need for expensive tests and physical prototypes, reducing the cost and increasing the speed of innovation. The cost of developing new drugs, for example, reaches into the billions, and preclinical testing phases alone take an average of three and a half years.

3. Innovating at the limits. When it comes to solving big human and social problems, the process of innovation becomes that much tougher. It may be unethical to run experimental tests on somebody's heart, for example, and one can't stop traffic in a city's rush hour to experiment with new routing systems.

\subsection{A Digital Twin's Journey}

In a White Paper entitled: 'Gaining Business Intelligence From Your Digital Transformation' the Vertexvis company (Vertexvis, 2019, p. 4) specified the value of a digital twin citing Gartner's opinion that 'digital twin is the top technology trend for the last three years running'.

The White paper contains short descriptions of applications of the concept in practice:

"Digital twins give context and insight into each stage of the product lifecycle, including design, build, and in-the-field usage.

1. Engineering and design teams create a product's design, including requirements and simulation results to understand how a product will perform. This "as-designed" information kicks off the digital twin's journey.

\footnotetext{
${ }^{2}$ Gartner Identifies Top 10 Strategic IoT Technologies and Trends, https://www.gartner.com/en/newsroom/pressreleases/2018-11-07-gartner-identifies-top-10-strategic-iot-technologies-and-trends.
} 
2. As manufacturing teams turn the design into reality, they quickly add any replacements or changes to the digital twin to record "as-built" information.

3. Once a product is in the field, a multitude of sensors gather information about the product's performance. This information streams back into the digital twin to record "as-manufactured" data.

4. Companies take design, build, and performance data to understand what is needed for "as-serviced" information and provide learning and predictive analytics. With the right technology in place, a digital twin can understand information from sensors and relate it to the as-designed and as-built information to gain insight on improving future iterations".

\subsection{Digital Twin Challenges}

In the opinion of experts of many industrial advisory companies efficiency in operations and product design optimization have become strategic imperatives in manufacturing. In addition, pressure from consumers, investors, and regulators to adopt sustainable business practices makes it more difficult for manufacturing companies to maintain their bottom line. Although digital twin technology is often marketed as the silver bullet for manufacturing, offering to increase inefficiencies in operations, facilitate innovative product design, and promote sustainable practices, digital twins are simply a tool. The reality is that obtaining the technology does not guarantee success - companies need to know how to effectively leverage it to achieve their sustainability goals. And successful implementation requires changes at the organizational level (Guidehouse Inc., 2019).

While digital twins have plenty of momentum, significant challenges remain. Technology providers haven't adopted a standard definition of a digital twin, and most describe the set of capabilities in slightly different ways. There's also the issue of data sovereignty — specifically, how to persuade customers and partners to share data collected from assets with manufacturers so they can improve the digital twin to the point it can support preventative maintenance services and optimize future product designs. What's also required is standardization of data management processes and clarity about the available tools to create and manage digital twins. While implementing digital twins doesn't require a whole new toolbox, it does require a new approach for managing and using data.

According to Rafael Go, senior research analyst at Navigant Research (Go, 2020), apart from technology there are also the usual organizational and cultural hurdles. There is still a lot of inertia that prevents manufacturing companies from doing things in different ways. Building organizational comfort and buy-in with the digital twin concept is important. Go considers that "the most important dimension pertaining to the organization is the breadth and depth of the information. The latest applications of digital twin technology require real-time data from a multitude of sources throughout the organization. Enabling timely and proper access and use of this data requires retooling the data sharing, security, and access approaches within the 
organization. Data use protocols and procedures would need to be re-examined and rewritten to break down siloes in data ownership and access while ensuring that sensitive and confidential data is protected. In addition to changing data management practices, companies also need to train their workers to become more data literate. Companies must invest in training and hiring programs that emphasize increasing employee comfort working with data. Taking full advantage of a technology such as the digital twin with organization-wide implication requires an enterprise wide approach".

\section{Blockchain can improve the concept of digital twins}

A digital twin is a concept that refers to the virtual replica of a physical asset, like luxury goods or artworks, in the digital realm, for example on a blockchain. This requires creating a digital identity for such objects, allowing for their digital existence alongside their physical one. The digital identity is generated thanks to traditional authentication solutions (such as near field communication - NFC, radio-frequency identification - RFID, visual recognition, etc.) and is first independent from the distributed ledger. Once created, the digital identity can be associated with a token and managed on a blockchain. This helps track physical assets and integrate them into dematerialised processes to optimise the operation and maintenance of the underlying physical assets. This also encourages innovative solutions that combine both blockchain and Internet of Things - IoT.

\subsection{Examples of case studies}

Case Study 1 (Blockchain forum.eu: report digital assets 2020a, p. 17): LVMH (Louis Vuitton Moët Hennessy) and the AURA platform. "LVMH is developing AURA, a platform that aims to serve the entire luxury industry with product tracking and tracing services on Ethereum $^{3}$. All along the value chain, from production to distribution, LVMH's luxury goods "live" on blockchain. Indeed, once one product is manufactured, a token is minted and affiliated with this product. When the product is sold, the related digital twin is transferred to the acquirer. Consumers can then access the product history and proof of authenticity of any of the items they buy, at any stage of their lifecycle. This is made possible by recording these attributes on the blockchain and attaching a certificate containing all the product information: details on product origin and components, instructions for product care, after-sales and warranty services available. At this stage, even if it is technically feasible, tokens are not traded on secondary markets".

\footnotetext{
${ }^{3}$ Blockchain glossary; https://comptiacdn.azureedge.net/webcontent/docs/default-source/research-reports/07576blockchain-glossary-of-terms-r3.pdf?sfvrsn=7df7462a_0.
} 
Case Study 2 (Blockchain forum.eu: report digital assets 2020a, p. 17): “TEO - The Energy Origin Engie, a French supplier of gas and electricity, and Ledger, a French blockchain startup known globally for its hardware wallets ${ }^{4}$, partnered to create The Energy Origin (TEO) platform. This project is based on several observations. First, climate change and energy transition are now compelling issues for energy suppliers, which are shifting towards more responsible production. Second, citizens have become increasingly concerned by their energy consumption and favour green power sources. However, it is very hard for them to measure the real impact of their efforts on the environment and to know the precise origin of the energy that they use. TEO records in real time both generated and consumed energy volumes thanks to sensors installed on sites. Based on the customer's preferences, TEO's matching engine calculates the volumes of energy exchanged between the renewable assets and the customer's sites as well as the impact in terms of avoided $\mathrm{CO} 2$. This information is registered in tamperproof certificates and accessible on TEO. Tokenising "avoided CO2" enables citizens to value their green commitment. Based on this first layer, further use cases could be imagined, such as a system of rewards or monetisation of energy savings".

\subsection{The future}

By 2020, it is expected that there will be 4.5 billion networked Internet of Things (IoT) devices in Europe, providing digital twins with the data they need (Miskinis, 2018). Blockchain has naturally become the next step in bridging the connection, with the advent of blockchain technology redefining the concept of digital twins. It could be an essential tool to aid the application of digital twins in IoT, transferring the data and value onto the Internet with full transparency. Sectors such as manufacturing and retail are set to benefit from these technologies interlinking, connecting the physical with the digital world. A digital twin built with traditional technologies must need a central intermediary that is dependable in performing analytics and holding data. Perhaps the inability to govern the digital twins and the lack of approval is a reason why digital twins have been slow to gain popularity. Blockchain technology has the potential to become the most relevant and capable way to generate and monitor digital twins due to the security and immutability of technology.

Platform security of the digital twin platform and data is crucial. Blockchain technology enables security from hackers due to its encryption features and provides data history transparency. Digital twins will benefit from these features, as it can transmit data securely. Both small and medium enterprises (SME) and large companies are beginning to utilize blockchain technology to store physical objects, and connecting digital twin technology will soon be the next step. In order for this to succeed, specialized distributed ledger platforms that allow sharing information amongst digital twins are needed. Digital twins on a blockchain will also help with brand protection and counterfeit items. Creating a digital twin of a product on

\footnotetext{
${ }^{4}$ Ibidem.
} 
blockchain would see its transaction records saved, along with information about where it was made, and its previous owners. In addition to this, for the first time in history companies who combine blockchain with virtual simulations will be able to track their product globally with extreme accuracy - this will totally eliminate the number of stolen goods across the globe (Miskinis, 2018). There is a strong belief (Purdy et al., 2020) that impact that digital twins can make is huge. Many are just now in development due to the complexity of their creation, but soon millions of things will have digital twins. And their usefulness and capabilities will continue to evolve. The digital twins of two - or more - of these robots can communicate to figure out how to best work together on a new assembly line.

But it doesn't stop there. Linking the digital twins of completely different types of assets can feed the digital twin of more complex entities. An entire city, for example, will need to connect information from digital twins tied to traffic, weather patterns, pollution, citizens, energy, and other resources. This enables a transformation from smart cities to conscious cities - cities that are aware of assets flowing in and out of their borders. This level of understanding unlocks new ways of innovation (Maxwell, 2018): new forms of urban mobility that are both faster and cheaper, elimination of congestion; reduction of pollution; new ways of emergency planning; and smart tracking of energy and water. A digital twin with all the complexity and detail of a physical city provides a larger and more valuable platform for experiments than has ever been created.

\section{Summary}

Nowadays it is obvious that the blockchain technology is revolutionising just about every industry imaginable. Banking, politics, healthcare... all could technically benefit from the creation of a decentralised digital ledger which tracks and stores information in various places, thus making forgery impossible. Identification is provided through complex calculations, making identity theft virtually impossible, too.

This general review paper provides an overview of some concepts and techniques concerning digital assets especially in the form of digital twins and presents opinions of different research circles on how, by applying the blockchain technology, the concept of digital twins can be improved. As a basic source of opinions served works of The European Blockchain Observatory and Forum which launched in February 2018, and which is a European initiative to accelerate blockchain innovation and the development of the blockchain ecosystem within the EU (EU Blockchain Forum Working Group; 2018). Even though the digital asset phenomenon is well under way, there is still a long way to go and obstacles to overcome before it becomes a real revolution. 
In the opinion of members of the Forum, "the European Union is currently focused more on the risks that arise from digital assets, to the detriment of the great opportunities that they bring. They think authorities should continue their efforts to ensure the healthy growth of digital assets in a safe environment for consumers and established players and take additional measures to help innovative actors progress, experiment and prove their positive potential for the European economy and markets. This starts with providing legal certainty for these actors, which actually means greater clarity for regulators to supervise the digital asset ecosystem" (Blockchain forum.eu: report digital assets 2020a, p. 33).

The final conclusion is the following: impact that digital twins can make is huge. Many are just now in development due to the complexity of their creation, but soon millions of things will have digital twins. And their usefulness and capabilities will continue to evolve. On the other hand, blockchain technology has the potential to become the most relevant and capable way to generate and monitor digital twins due to the security and immutability of technology.

\section{References}

1. Blockchain forum.eu: Blockchain and the future of digital assets, report (2020a). Available online: https://www.eublockchainforum.eu/sites/default/files/report_digital_assets_v1.0. pdf? width $=1024 \&$ height $=800$ \&iframe $=$ true, 3.08.2020.

2. Blockchain forum.eu: Blockchain and the future of digital assets; Blockchain terminology, report (2020b). Available online: https://www.eublockchainforum.eu/sites/default/files/ report_digital_assets_v1.0.pdf?width=1024\&height=800\&iframe=true, 3.08.2020.

3. Blockchain Research Institute (BRI) (2020). Available online: www.blockchainresearchinstitute.org.

4. CompTIA.org: The Role of Emerging Technology in Digital Transformation, Whitepaper 2020. Available online: https://comptiacdn.azureedge.net/webcontent/docs/defaultsource/research-reports/07821-emerging-tech-whitepaper-online.pdf?sfvrsn=a0cbca08_0, 13.08.2020.

5. CompTIA.org: Using Strategic IT for Competitive Advantage, Whitepaper 2018. Available online: https:/comptiacdn.azureedge.net/webcontent/docs/default-source/research-reports/ using-strategic-it-for-competitive-advantage.pdf?sfvrsn=9b963e69_0\%22, 13.08.2020.

6. CompTIA: A Functional IT Framework, Report 2016. Available online: https://comptiacdn.azureedge.net/webcontent/docs/default-source/research-reports/02878it-framework-report-online.pdf?sf, 13.08.2020. 
7. EU Blockchain Forum Working Group; 2018. Available online: https://www.eublockchainforum.eu/news/eu-blockchain-observatory-and-forum-namesmembers-core-working-groups, 3.08.2020.

8. Gaining Business Intelligence From Your Digital Transformation, White Paper (2019, p. 4) Vertexvis company. Available online: https://vertexvis.my.salesforce.com/sfc/p/\#f 4000002c0zl/a/f4000000cKVK/QBbA3.ennNRNidwlbV4CjF7wOOyynxi89Sn2dE8e39o, 10.09.2020.

9. Gartner (2018). Top 10 Strategic IoT Technologies and Trends. Available online: https://www.gartner.com/en/newsroom/press-releases/2018-11-07-gartner-identifies-top10-strategic-iot-technologies-and-trends, 10.09.2020.

10. Go, R. (2020). Organizational Barriers to Harnessing the Digital Twin. Guidehouse Inc. Available online: https:/guidehouseinsights.com/news-and-views/organizational-barriersto-harnessing-the-digital-twin, 10.09.2020.

11. Leveraging Digital Twin Approach for Sustainable Manufacturing. Guidehouse Inc. (2019). Available online: https://guidehouseinsights.com/reports/leveraging-digital-twinapproach-for-sustainable-manufacturing, 10.09.2020.

12. Maxwell, L. (2018). Smart city benefits: How smart cities save governments, businesses and citizens money; Bee Smart City. Available online: https://hub.beesmart.city/en/strategy/ how-smart-cities-save-governments-businesses-citizens-money.

13. Miskinis, C. (2018). How the blockchain can improve the concept of digital twins; Challenge Advisory. Available online: https://www.challenge.org/insights/blockchain-anddigital-twin/, 10.09.2020.

14. Purdy, M., Eitel-Porter, R., Krüger, R. and Deblaere, T. (2020). How Digital Twins Are Reinventing Innovation. MIT Sloan Management Review. Available online: https://sloanreview.mit.edu/article/how-digital-twins-are-reinventing-innovation/ ?gclid=Cj0KCQjw7sz6BRDYARIsAPHzrNKv16P8zykbiuK7JAdESbMiLZln3g_Ly51Xk 7mpfPND2VojPdn9HZoaArLxEALw_wcB, 10.09.2020.

15. Tapscott, D. (2020). Token Taxonomy. The Need for Open-Source Standards Around Digital Assets. Toronto: Blockchain Research Institute. Available online: https://www.blockchainresearchinstitute.org/wp-content/uploads/2020/02/Tapscott_ Token-Economy_Blockchain-Research-Institute.pdf?utm_source=hs_email\&utm_ medium $=$ email\&utm_content $=84603290 \&$ hsenc $=$ p2ANqtz-8_5Yo_tQVzMnAbgg CBV8fxDhF3uSOgs1HpyISLd6FGH0zMTvp6QFnuH_2I376r6WhherbIZWdVj6x6mSFJ TzRe5QWvFg\&_hsmi=84603290, 20.08.2020. 